\title{
On the physiological basis of masking with dotted visual noise ${ }^{1}$
}

\author{
WILLIAM R. UTTAL ${ }^{2}$ \\ THE UNIVERSITY OF MICHIGAN
}

In this paper, consideration is given to the physiological foundations of visual masking with successive stimuli. Three experiments are performed with dot patterns. The results indicate that none of the classic characteristics of lateral inhibitory interaction are obtained with this type of stimulus material. A rather informal demonstration is then described that suggests further that lateral inhibitory interaction may also not be a satisfactory explanation of metacontrast and other kinds of masking that use continuous figures.

Studies of visual masking (see general reviews by Raab, 1963; Kahneman, 1968; and Weisstein, 1968) typically can be classified into three different kinds of experimental paradigms. The first category involves the use of a very bright flash of diffuse light that completely overlaps the test stimulus as a mask. The second category uses such geometrical, but nonoverlapping, test and masking stimuli as adjacent rectangles (Alpern, 1953; Kahneman, 1967) or such nearly identical contours as a disk and annular ring (Kolers \& Rosner, 1960; Schiller \& Smith, 1966). Experiments of the third category use random patterns of visual stimuli as a mask for some geometrical form. Typically, the component parts of the masking noise and the test stimulus are the same, and the noise and test stimulus usually are designed to overlap. Reports by Kinsbourne and Warrington (1962a, b) and by Schiller (1965) fall into this category.

In on of the earlier papers in the present series (Uttal, 1969a), it was suggested that the effects of several different, potentially distinguishable masking processes might be mixed together in many of the reported experiments. It was also pointed out that when one is using patterns of dots as the visual noise, there is reason to believe that different mechanisms may underlie the masking effects than in the cases of the other two categories. Dots positioned at random locations and presented at moderate speeds are unlikely to stimulate the same retinal loci successively, and thus, the general dark adaptation level of individual receptors remains relatively constant. This hypothesis also implies that photochemical interactions and inertia in the receptors are not likely candidates for explanations of many of the effects. Dot patterns do not have continuous contours in the same way a ring, disk, or rectangle does, other than those organizational ones that appear only in the "eye of the beholder."

In this paper, we shall concern ourselves mainly with the physiological basis that underlies this special case of the third kind of masking-masking with random dot patterns. Many recent workers (Weisstein, 1968; Rothberg, 1968; Buchsbaum \& Mayzner, 1969; Parlee, 1969; Dember \& Purcell, 1967; Robinson, 1968; Mayzner, Tresselt, \& Helfer, 1967) as well as an older paper (Alpern, 1953) have turned towards the powerful and well known mechanism of lateral inhibitory interaction as an explanation of the effects obtained in other forms of visual masking.

The question we ask in the present paper concerns whether or not lateral inhibitory interaction can underlie the effects obtained with dotted masking noise. The conclusion that has been reached is that it does not, and furthermore that, in retrospect, serious questions can be raised concerning the applicability of this physiological mechanism to the other kinds of visual masking as well.

The basic physiological properties of lateral inhibitory interaction in peripheral nerve nets are well understood (von Békésy, 1967; Ratliff, 1965). A formidable and conclusive body of evidence (e.g., Hartline \& Ratliff, 1957, 1958) has been presented linking this physiological mechanism to such spatial percepts as the Mach band, the checkerboard illusion, and similar spatial patterns. However, the body of knowledge that supports the application of this idea to temporal patterns is not so complete. In fact, as we shall see, evidence seems to be accumulating to the effect that masking situations are not reflected by single cells (Schiller, 1968), and that dots do not exhibit lateral inhibiting properties (von Békésy, 1968). Furthermore, the extensive research with identical rectangles and similar disks and rings obscures the fact that during the early days of this kind of research, considerable difficulty was encountered in finding any particular stimulus shapes that exhibited the phenomenon, and that, in general, these masking stimuli are examples of a relatively rare phenomenon. Masking is, as we shall show in our fourth experiment, highly form-specific. Others have shown that as simple a manipulation as lengthening one of two lines that had previously masked each other when they were the same length can result in a diminution of the masking effect (Buchsbaum \& Mayzner, 1969). Similarly, changing the angle of the masked line to some other inclination can reduce the masking effect (Parlee, 1969; Sekuler, 1965). Thus, masking effects are seen to be dependent upon form similarities in a way that suggests that lateral inhibitory interaction in the peripheral receptor plexus, as we usually know it, cannot be directly applied in general as an explanation of the masking effect. Rather, some more central or "cognitive" mechanism will probably have to be invoked to explain many of the masking results.

In the present paper, we concentrate on data that refer specifically to masking with dots. The results of the first experiment show that no masking exists when the stimulus and the mask do not overlap, confirming Kinsbourne and Warrington's (1962a) results with continuous figures. In the second experiment, it is shown that the "disinhibition" phenomenon reported by Robinson $(1966,1968)$ and replicated by Dember and Purcell (1967) for rings, disks, and solid letters also does not obtain with "dotted noise." In the third experiment, by performing the dichoptic experiment (test character to one eye, dotted noise to the other), we demonstrate that, at the very least, the decrease in recognition scores that occurs with masking with dots is not mainly linked to retinal effects but involves some higher level of the nervous system. This extends to dot patterns in the earlier work on dichoptic masking by Kinsbourne and Warrington (1962b), Gilinsky and Doherty (1969), and Kolers and Rosner (1960). All three of the present experiments are done with static dot patterns in which a large number of dots are plotted as fast as possible in random locations across a specific region on the face of the oscilloscope. This technique differs in a critical way from the work on dynamic visual noise (DVN) that we have reported earlier (Uttal, 1969a, b, $1970 a, b)$, wherein a dynamic pattern of randomly located dots was displayed over an extended period of time on the face of the oscilloscope. The present technique thus comes much closer to that originated 
by Eriksen and Collins (1967), in which sequentially presented patterns of dots were combined to make recognizable figures. However, rather than leading to the emergence of a formed perception, our technique seduces the recognizability of an alphabetic character set.

Finally, we carried out a rather informal experiment that speaks directly to the point that form specificity is necessary for masking effects to occur. This experiment is done not with dotted and randomly placed noise, but with an oscillographic display that more closely approximates the continuous line drawings used in the conventional tachistoscope.

\section{Subjects}

\section{GENERAL METHOD}

For the first three experiments, a group of eight undergraduate students at the University of Michigan participated for a complete semester and were paid hourly wages. Each $S$ was given a 4-day pretraining session with slight amounts of interfering noise, before data collection began, to familiarize him with the alphabetical font used in the experiment. All eight Ss participated in the first three experiments, and each data point plotted in our figures is the pooled result of all responses of all Ss. In the last experiment, a random selection of Ss was chosen by canvassing volunteers for about $15 \mathrm{~min}$ of participation each.

\section{Apparatus}

In all of the four experiments we report in this paper, the Ss were seated in a soundand light-tight acoustical booth. Their heads were not constrained other than by means of a forehead rest placed a fixed $14 \mathrm{in}$. in front of the screen of the cathode-ray tube. This CRT was surfaced with a special ultrashort persistence (P-15) phosphor on which light output will decay to about $0.1 \%$ of its original brightness in about 50 microsec. The position of a pattern of dots was determined by selecting the horizontal and vertical coordinates of sequential locations under the control of a digital computer. In some experiments and in some parts of all experiments, patterns were generated by plotting dots in the positions defined by table entries. In this way, fixed figures or alphabetic characters could be generated. The alphabetic characters were generated

Fig. 1. A diagram of the stimulus configuration used in Experiment 1. A character formed from dots is placed within a "masking" stimulus region composed of similar dots. Masking dots are excluded from the central region in which the character appears. by selection of a pattern of dots from a 5 by 7 dot matrix in this manner. Some of the figures in the fourth experiment, however, contained a considerably larger number of dots to define an almost continous figure.

In the other three experiments in which random dot patterns were used as masks, random number algorithms in the computer were evaluated prior to the running of each trial to fill up a table specifying random dot locations. Then the patterns were plotted as rapidly as possible by sequencing through this list. In this manner, 100 dots in random locations could be plotted in less than $3 \mathrm{msec}$. Because of the persistence of the visual process, the appearance of this burst of dots to the $S$ was that of a briefly flashed random array of dots, all of which appeared and disappeared simultaneously. Various subroutines were used as necessary to alter the time of occurrence relative to the masked stimulus or to exclude certain regions of the area from plotting dots.

The alphabetic character set was the same as that used in earlier experiments. It was composed of 24 characters (omitting $O$ and $Q$ for obvious reasons) which were slightly smaller ( 4 by $3 \mathrm{deg}$ ) than the region covered by the static noise pattern ( 9 by 6 deg).

In the first three experiments, two Ss ran as a team. Each $S$ spent $1 / 2 h$ in the experimental cubicle as the $O$, and then changed places with his partner who had been transcribing the data into the computer by means of a typewriter-like keyboard. The actual character presented and the character which the $O$ reported were both displayed on another television screen in front of the transcriber. He relayed the correct character to the observer S when, and only when, a mistake had been made. All communication between Ss was accomplished by using a phonetic alphabet to reduce dictation errors.

Data were collected on digital magnetic tapes and were analyzed at the end of each day by appropriate analysis programs. In general, results of about 2,500 trials for all eight Ss were pooled for each data point plotted on our graphs, but in some of the more difficult conditions, fewer trials occurred as the $S$ slowed his pace.

A dim fixation point appeared on the CRT in front of the observer $S$ when the computer was ready for the next trial. He then depressed a hand-held switch, and the sequence of stimuli was released by the computer. We shall describe what the specific stimulus conditions were in the following discussions of the individual experiments.

On each day, the independent variable of each experiment was held constant, but the alphabetic character set was presented in a purely random order as defined by a multiplicative random number program evaluated by the computer.

\section{Method}

\section{EXPERIMENT 1}

Experiment 1 was designed to determine if nonoverlapping but surrounding

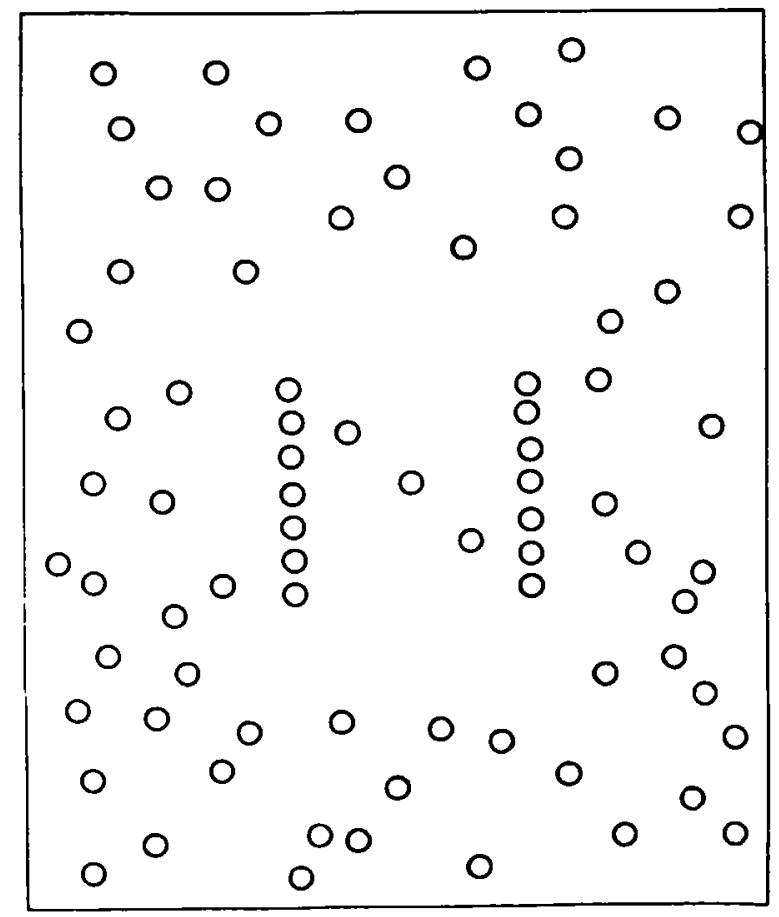


random-patterned masking dots could inhibit the recognition of dotted alphabetic characters. Figure 1 is a diagram of the appearance of the random visual noise pattern and the character. The visual noise was excluded from a rectangular region around the character, with dimensions of about $41 / 2$ by $31 / 2 \mathrm{deg}$.

In this experiment, the independent variable was the interval between the presentation of a character and the presentation of a burst of 100 dots randomly dispersed in the region surrounding the character position (but, as shown in Fig. 1, excluding the central region in which the character appeared). Intervals of $100,75,50,35,25,15$, and $0 \mathrm{msec}$ between a leading character and the trailing noise burst were used. The dependent measure was the percentage of the total number of presented alphabetic characters that was identified correctly.

\section{Results}

The results of this experiment are presented in Fig. 2. It can be seen that throughout the entire range of intervals used, there was no masking effect of the surrounding pattern of masking dots on the recognition of the characters. This range of intervals includes the peak inhibiting interval of $80 \mathrm{msec}$ found in the usual conventional metacontrast experiment using a disk and annular ring (Kolers \& Rosner, 1960) or three rectangles (Kahneman, 1967) as well as the region $(0-35 \mathrm{msec})$ in which we have previously found high degrees of masking for overlapping DVN patterns (Uttal, 1969a). Thus, we may conclude, as did Kinsbourne and Warrington (1963a) with continuous figures, that masking with noise is only effective when the test and masking stimuli actually overlap in the same visual area.

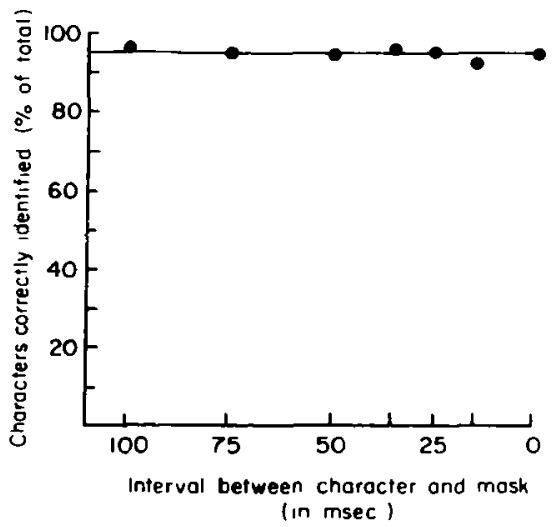

Fig. 2. The results of the first experiment. Over 100 -msec range, there is little evidence of any masking effect by the nonoverlapping noise pattern.

\section{EXPERIMENT 2}

Method

In Experiment 2, we attempted to detect the effects of a "disinhibiting" stimulus on the recognizability of alphabetic characters that were masked by a burst of trailing dots. "Disinhibition," a term used by Hartline and Ratliff (1957), refers to the increase in detectability or recognizability of a stimulus when a third stimulus acts to mask or inhibit a second masking stimulus. The effects of the original masking stimulus on a test stimulus are thus lessened by the disinhibiting stimulus, and the test stimulus is more easily seen.

This experiment was composed of two parts. In the first part, which has been reported earlier in a different contex (Uttal, 1970b), the presentation of the character was followed by a single burst, with the interval before the burst serving as the independent variable. Intervals of 100 $75,50,40,30,20,10$, and $0 \mathrm{msec}$ were used. Inhibition of character recognition was the dependent variable and, as usual, was employed as an assay technique to ineasure the relative effects of the various parameters. In the first part, the number of dots in the following burst was always 100 . Once these data were at hand, it was possible to select an appropriate value for the delay between the character and the first burst as a standard for the second part of the experiment.

The second part of the experiment was the disinhibition study per se. Here we added a second burst of 100 dots at various delays following the first inhibiting or masking burst. The second delay between the inhibitory and potentially disinhibitory stimuli had values of $20,30,40,50,75$, and $100 \mathrm{msec}$. The first delay between the character and the first burst of masking dots was kept constant at 20 mses. If the situation with dot patterns was directly comparable to the ring and disk experiments described above, then a similar sort of disinhibition should have occurred, and an increase in the percentage of the recognized characters should have been observed.

\section{Results}

The results of both parts of this experiment are shown in Fig. 3. The declining curve shows the effect of varying the separation between the dotted character and a single following burst of interfering dots. Recognizability of the character, as reported earlier, is seen to decrease the closer the dots are to the character. The results of the second part of the experiment, in which the first delay was kept constant at $20 \mathrm{msec}$ and the second delay was varied, are seen to be

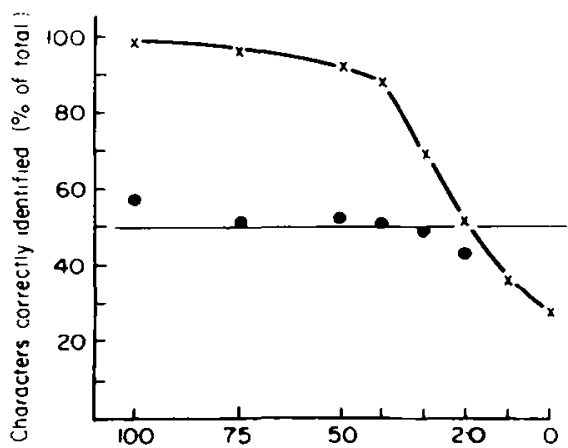

$x=$ Interval between choracter and first noist burst (in msec)

- Interval between first and second noise bursts (in $\mathrm{msec})$

Fig. 3. The results of the two parts of Experiment 2. The gradually descending curve shows the effect of a single noise burst at various delays following the character. The horizontal flat curve shows the results (for a 20 -msec interval between the first noise burst and the character) when a second noise burst is presented at various intervals following the first noise burst. Little effect is seen on the masking when the second burst is present, except for the further decrease in performance at the 20-msec interval and a slight increase in performance at 100 msec. This latter point is considered outside the disinhibition range reported previously and is not believed to be related to the disinhibition phenomenon.

fitted with a horizontal line. Deviations from the control performance level are slight and most pronounced for the shortest value of the second delay. The change in this case is a decrease rather than an increase, indicating that the masking effects of the two bursts following the character are increasing rather than diminishing each other. From the first part of the experiment, it is clear that the dots of the second burst ( $20 \mathrm{msec}$ after the first burst; $40 \mathrm{msec}$ following the character) would have some small inhibitory effect themselves. Apparently, that additional effect has been added to the effects of the first burst to cause the slight decline of this point.

An equally slight increase in the percentage of characters recognized correctly is observed at the longest interval used, but this delay is so far outside the range of intervals usually reported for disinhibition that we do not believe it to be significant in the context of the current discussion. The general result of this experiment is that there is no disinhibition of the kind previously reported in the range for other kinds of backward-masking stimuli. This is contrary to the results 
reported by Robinson $(1966,1968)$ and by Dember and Purcell (1967).

\section{EXPERIMENT 3}

Method

The purpose of the third experiment was to determine whether or not the effects previously measured binocularly could be obtained dichoptically. All the papers cited above that report dichoptic masking imply that the interaction leading to masking is not retinal and must necessarily occur at higher levels of the nervous system, where the existence of lateral inhibitory interaction is not equally well established. Our current technique is sufficiently precise to determine whether the effect is completely explained on the basis of binocular fusion or if, on the other hand, there is some difference in the dichoptic, binocular, and monoptic situations.

This experiment was run in three parts. In the first part, the dichoptic situation was examined. $S$ viewed the face of a split-screen CRT through rotary convergence-controlling prisms. An opaque septum divided the screen so that the left eye could only see the left side of the screen and the right eye only the right side. The right prism only was individually adjusted so that two tiny fixation points were fused comfortably for each $S$. When this fusion was complete and steady, the $S$ pressed the hand-held control switch and released the stimuli. The stimuli were presented in the following manner: The character was plotted on one side of the screen and then, immediately following, the burst of noise on the other. Prior to the display of the character and the burst of dotted noise, a one-bit random number generator had automatically selected whether the character would be on the right or left side. In this manner, the $S$ was prevented from closing one eye and from either consciously or by some subtle attentional manipulation occluding the noise.

The independent variable used in all three parts of the third experiment was the number of dots in the masking burst. Numbers of dots were: $1,20,40,60,80$, and 100 .

In the second part of the experiment, the conditions of the first experiment were repeated monoptically to provide a standard against which to compare the results of the binocular part of Experiment 3. The $S$ continued viewing through the rotary prisms, but the computer was reprogrammed to present the character and the burst of noise superimposed in the field of view of only the right eye. The right prism was set to a 6-deg angle of convergence to mimic the optical condition of the first experiment.
In the third part of the experiment, the binocular condition was run in which both eyes saw both the character and the dotted noise, which were actually superimposed. The S still observed the stimuli through the rotary prisms to control for any optical artifacts they might have introduced. Again, the right-hand prism was set to $6 \mathrm{deg}$ to mimic the optical conditions of the first part of this experiment.

\section{Results}

The results of the three parts of Experiment 3 are presented in Fig. 4. This figure indicates that presenting the masking and test stimuli dichoptically has less of a masking effect than having both presented to a single eye. Furthermore, when the overlapping stimuli and noise are observed binocularly, the results are very close to those for the monoptic case, indicating that there is no particular advantage to binocular viewing, and that the very fact that the information was presented monoptically did not, in some subtle way unrelated to our main experimental variable, reduce the S's performance level. Thus, we may conclude from the three parts of this experiment that the presence of noise and character dots on the same peripheral portions of the photoreceptor does introduce a slight additional masking effect, in addition to the major masking effect mediated by some more central mechanism where the binocular fusion occurs. $^{3}$

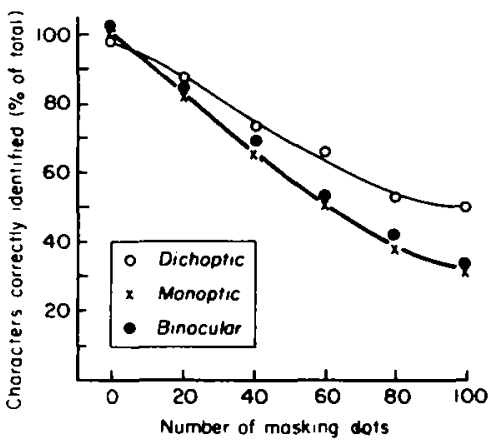

Fig. 4. The results of the three parts of Experiment 3. The three curves are for the dichoptic, monoptic, and binocular conditions. The monoptic and binocular conditions are seen to produce a somewhat greater masking effect than the dichoptic. Since the former curves do not differ significantly from each other, this suggests that there may be some lesser peripheral masking effect independent of monocular-binocular viewing conditions. However, the major masking effect appears to be mediated centrally.

\section{EXPERIMENT 4}

Method

Experiment 4 was run as a set of informal observations rather than as a formally designed study. Its purpose was to illustrate the high degree of form specificity that is required to produce some of the classic metacontrast (backward-masking) effects. The stimulus patterns were plotted on the CRT used for the other experiments, but rather than dot patterns, the traditional metacontrast stimulus pattern of three figures arranged along a horizontal axis was used. A test pattern was always flanked by two masking figures which followed at a controlled delay. These figures were formed by plotting dots very densely, and at normal viewing distances (about 36 in.), the dotted line appeared to be continuous. When the three figures are identical rectangles, the backward-masking or metacontrast effect is very strong when the delay is between 30 and $100 \mathrm{msec}$ in duration. The dynamics of this phenomenon have been reported in detail by Alpern (1952) and Kahneman (1967). In the present experiment, the delay between the test and masking stimulus was kept constant throughout at $80 \mathrm{msec}$. The experimental variable was the shape of the central figure or flanking figures. The complete set of patterns used is shown in Fig. 5.

\section{Results}

The results of this demonstration were clear-cut. When the center figure was identical to the flanking figures, there was a very high degree of metacontrast and many reports of "I did not see it" or incorrect descriptions eventuated. When the center figure was changed in size or altered to one of the other test forms, but flanked by rectangles, errors of recognition were far less frequent. This occurred even when the figures used were parts of, or included parts of, the rectangle, such as the two bow-tie orientations.

On the other hand, when the center figure was a triangle and was flanked by two triangles, the masking effect was as strong as with three rectangles. Thus, recognition of the form seems to be a critical factor in the masking phenomenon, a result suggesting cognitive effects far more complicated than simple lateral inhibitory interaction.

\section{DISCUSSION}

The main question to which this paper is addressed concerns the physiological foundations of the kind of masking that occurs when dot patterns interfere, with the recognition of other dot patterns. Our general approach in attempting to answer this physiological question has been 
a.<smiles>C1CCC1</smiles>
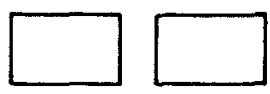

b.
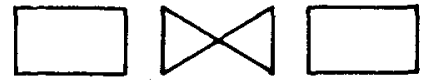

C.
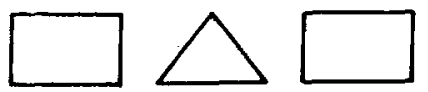

$d$
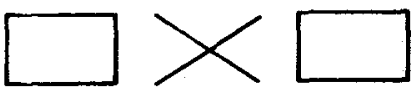

e.
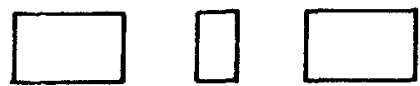

f. $\square$
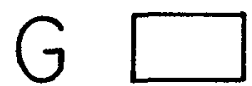

9
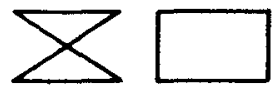

indirect. We have focused on relevant psychological studies rather than on surgery or microelectrode probing. Certain functional properties are well established from direct physiological observations of lateral inhibitory anatomical mechanisms. It is on the basis of the presence or absence of these functional properties that conclusions have been drawn concerning the pertinence of this specific physiological mechanism in the dot-masking paradigm. A second and related issue concerns whether the physiological mechanisms underlying masking with dots and masking with continuous forms are identical or different.

First, we concern ourselves with the mechanisms of masking with dot patterns. It is well known that lateral inhibitory interaction can occur when visual stimuli are not overlapping (Hartline \& Ratliff, 1957). Thus, regions of activity near but separate from each other can reciprocally reduce each other's responses when both are activated, even though stimulus conditions on each one are held constant. The characteristic lack of a requirement for overlap is shared by lateral inhibitory interaction measured neurophysiologically and by psychophysical tests with continuous forms, but the results of Experiment 1 show a sharply different result. The dot patterns do not affect the recognition of the characters unless they are actually superimposed. This experiment, confirming Kinsbourne and Warrington's (1962a) results with block characters, therefore begins to suggest that h.
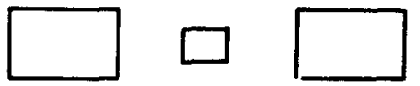

i
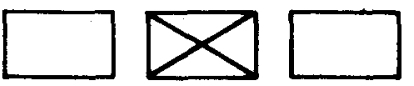

i.
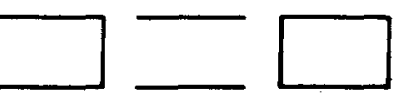

K.

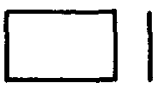

1

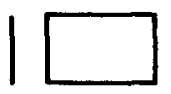

1
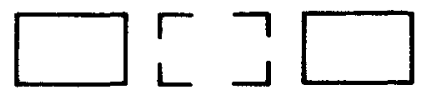

$m$.
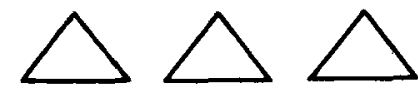

lateral inhibitory interaction is not a contributing factor in this kind of visual masking. It is also important to note that the overlap that is necessary to produce masking with dots may be introduced by either physically superimposing the figure and the noise dots in the same stimulus space or by physiologically mixing them through dichoptic fusion, as we have done in Experiment 3.

A second general mechanism that has been shown to be usually characteristic of lateral inhibitory interactive mechanisms is the phenomenon of disinhibition-the diminution of the inhibitory effect of a mask on target detection by a secund mask that apparently inhibits the first mask. The results of our second experiment, again, do not show any such second-order masking with dot patterns over the range usually reported as the one including the disinhibition phenomenon. However, disinhibition is reported for the continuous figures typically used in related oxperiments (Dember \& Purcell, 1967; Robinson, 1966). Thus, in this case, we see not only an additional difference in the reported results for masking with dot patterns and masking with continuous forms, but also a difference in the results that suggests that one of the usual features of lateral inhibitory interactiondisinhibition-does not obtain with dot patterns.

The third test of lateral inhibitory interaction is a less specific one. It deals not with the presence or absence of lateral
Fig. 5. The various combinations of figures used in Experiment 4 to test the effect of form specificity on backward masking.

inhibitory interaction mechanisms in particular, but rather with whether or not such an interaction is central or peripheral. This is the test applied in our third experiment, where it was shown that the masking effect of dots is strong when the character and the noise dots are presented dichoptically. This result suggests that the major portion of the interaction between the signal dots of the character and the noise dots of the random pattern does occur more centrally than in the retina, at some level of the nervous system of a far more complicated biology than that of a retinal nerve net. However, it is also important to point out that there was a difference between the monoptic and binocular cases on the one hand and the dichoptic case on the other. Both monoptic and binocular cases showed nearly equal additional masking when compared to the dichoptic condition. The correspondence between the results of the monoptic and binocular cases suggests that there was no special bias introduced into our results when the $S$ observed with only one eye, but that the mixture of the stimulus dots and the mask dots in the same part of the peripheral visual system was the specific necessary condition for this additional masking effect. Whether or not this small peripheral effect is the result of a lateral inhibitory interaction as usually defined, however, cannot be resolved from our current experiments. It is also interesting to note that Schiller (1965) observed that the monoptic experimental design resulted in a somewhat lower masking effect than did the fused binocular configuration, with his patterned and continuous figure stimuli. Thus, we may conclude that the major portions of the masking effects that are obtained in the current study with dots do not seem to be dependent upon processes that exhibit the usually accepted functional characteristics of lateral inhibitory interaction.

Let us now consider the related issue concerning how applicable the lateral inhibitory interaction model is to the general class of experiments that includes metacontrast, backward and forward masking, and other related forms of temporal suppressions by sequential visual stimuli.

The main impact of our fourth experiment, of course, is not just that the form specificity that is seemingly required to produce masking effects precludes simple network models of masking. Furthermore, it suggests that processes at 
the highest levels of cognitive function are also involved in the masking effects found with the more usual continuous stimulus patterns as well as with the dot patterns. Neither stimuli that are parts of rectangles nor rectangles with additional features seemed to be inhibited in the way identical forms are. Nor is one part of a figure, such as the vertical sides of a middle rectangle, masked more than the horizontal sides, even though the former are closer to a corresponding part of the masking rectangle. We have already cited the work done with parallel lines (Parlee, 1969; Buchsbaum \& Mayzner, 1969) which shows that both nearly identical line length and orientation are necessary for maximum masking effects. It thus seems that masking effects with the conventional ring and disk and rectangular stimuli are also dependent upon prior recognition of the forms themselves rather than upon any simple network-like interaction in the periphery. Sequential stimulation masking effects may thus be far more complex phenomena than the proponents of the lateral inhibitory interaction model would lead us to believe.

We can also tum to some recent physiological evidence which tends to reduce the credibility of the notion of lateral inhibitory interaction as the basic foundation of sequential masking effects in general. Schiller (1968), pursuing his series of studies on the masking effects in an elegant microelectrode study of masking-like stimulus conditions, recording from the lateral nucleus of the cat, shows that inhibition of the first response by a second stimulus occurs only when the stimuli fall in the same receptive field. When the two stimuli do not overlap receptive fields, there is no physiological evidence of a depressed response. In an earlier paper, Schiller and Chorover (1966) also report that no evidence of suppressed evoked brain potential response is found when the stimuli do not overlap, but that suppression does occur when they do. Similarly, where Fehmi, Adkins, and Lindsley (1969) find suppression of the neural response at levels as low as the optic nerve or where Donchin, Wicke, and Lindsley (1963) find suppression of the evoked potential, it is always in situations in which there is actually optical overlap. In both these studies, the masking stimulus is always a bright and diffuse flash fully overlapping the field of a geometric test shape.

Thus, as a general result, reduction of the neural trace of a stimulus that does exhibit masking properties in analogous psychophysical situations does not occur unless there is specific overlap of mask and masked stimuli. So far, no neurophysiological experiment has shown a correlate of the sort of masking found with the disk and ring or with similar rectangle stimulus configurations. In fact, if the results of these experiments are substantiated and replicated and added to the implications of our present fourth experiment, a statement even stronger than a simple rejection of the lateral inhibitory interaction model may be made. This statement is that the masking effects with nonoverlapping stimuli are not due to reduction in the amplitude of the afferent signal intensity or information content but must be mainly due to interpretations by some central cognitive mechanism of relatively constant input.

Psychophysical evidence that diminishes the strength of the hypothesis that peripheral lateral inhibitory interaction is a major cause of the masking effects includes the dichoptic studies reported in this present paper as well as the similar positive results obtained in dichoptic experiments with different stimuli, reported by Kinsbourne and Warrington (1962a), Kolers and Rosner (1960), and Schiller (1965), among others. In the same vein, Kahneman (1967) also points out that the backward-masking effects that are so often associated with continuous nonoverlapping forms are highly labile responses that tend to depend very much on the criterion or set of the $O$. Thus, a simple neurophysiological model of masking effects is difficult to justify. Furthermore, von Békésy (1968) deals directly with the problem of point stimuli as potential inhibitors for other stimuli and concludes that there is, in fact, little evidence for any kind of inhibitory interaction being exerted by points, either on each other or on larger visual stimuli.

Quite to the contrary, a very strong argument for lateral inhibitory interaction is the precise prediction made by Weisstein's model (1968) of much of the previously reported data. Her work deserves the most careful consideration. The model she proposes is based primarily on the U-shaped curves reported over the years by several experimenters. However, Eriksen and Marshall (1969) have recently reported a failure to replicate the U-shaped function and, like Kahneman (1967), feel that the U-shaped functions may be a reflection of "variable criteria," a phrase that may easily be read to mean central cognitive functions resulting from highly complex and little understood neural interactions at levels vastly different from the simple peripheral biological interactions invoked so often by so many current workers in the field. In Weisstein's model, as effective as its predictions are, it is important to distinguish between the mathematical functions describing the psychophysical data and the neurophysiological assumptions that lead to those functions. The functions do turn out to be effective descriptors and good analogues of the perceptual data. It is very possible, however, that they might do so with little real association with the physiological mechanisms implicated by Weisstein. But this is a general criticism of mathematical theorizing rather than a specific critique of her ingenious theory. Nevertheless, there are too many physiological and psychophysical results that suggest that lateral inhibitory interaction might not be an adequate enough explanation of the wide variety of sequential masking effects to simply accept Weisstein's physiological assumptions, no matter how precise her predictions. At the simplest level, it would be most difficult for such a simple biological network theory to explain the gross shape (i.e., the difference between the U-shaped and monotonic shapes) of the curves resulting from experiments with different psychophysical procedures.

It is, therefore, our specific conclusion that masking with random dot patterns has little to do with the often-expressed notion that lateral inhibitory interaction underlies masking. We also join those other workers (Eriksen \& Marshall, 1969; Schiller \& Smith, 1966; Schiller, 1968; and Kahneman, 1967) who either implicitly or explicitly have suggested that a simplistic form of a laterally inhibitory nerve net model cannot explain the very complicated and interesting sequential visual masking effects.

With lateral inhibitory interaction thus challenged, and with stimulus overtake ruled out because of the identical luminance levels of the stimulus and the mask, the most likely overall explanation of the masking effects we obtain with dot patterns seems to be one in which the contours and organizational simplicity of the character are degraded by the visual noise. This occurs in the form of a simple mixture due to the persistence of the visual images. This is in line with Kinsbourne and Warrington's (1962a) notion and with Eriksen and Collins's (1968) comments on sensory traces. Thus, the results we present become closely analogous to the work on signal-to-noise ratios and signal detection in the auditory system and provide another new manner of studying the information-processing capability of the central human visual system in a manner that is independent of peripheral photochemical or neural interactions of secondary interest.

ALPERN, M. Metacontrast. Journal of the 
Optical Society of America, 195?, 43, 648-657.

BUCHSBAUM, W. H., \& MAYZNFR, M. S. The effects of linc length on sequential blanking. Psychonomic Science, 1968, 15, 111.112.

DEMBER, W. N., \& PURCELI., D. G. Recovery of masked visual targets by inhibition of the masking stimulus. Science, 1967, 157, 1335-1336.

DONCHIN, E., WICKE, J. D., \& LINDSLFY, D. B. Cortical evoked potentials and perception of paired flashes. Science, 1963, 141. 1285-1286.

ERIKSEN, C. W., \& COLlLINS, J. F. Some temporal characteristics of visual pattern perception. Journal of Experimental Psychology, 1967, 74, 476-484.

ERIKSEN, C. W., \& COLLINS, J. F. Sensory traces, versus the psychological moment in the temporal organization of form. Journal of Experimental Psychology, 1968, 77, 376-382.

ERIKSEN, C. W., \& MARSHALL, P. H. Failure to replicate a reported $U$-shaped visual masking function. Psychonomic Science, 1969, 15. 195-196.

FEHMI, L. G., ADKINS, J. W., \& LINDSLEY, D. B. Electrophysiological correlates of visual perceptual masking in monkeys. Experimental Brain Research, 1969, 7, 299-316.

GILINSKY, A., \& DOHERTY, R. S. Interocular transfer of orientational effects. Science, 1969 , $164,454-455$.

HAR TLINE, H. K., \& RATLIFF, F. Inhibitory interaction of receptor units in the eye of the Limulus. Journal of General Physiology, 1957. 40, 357-376.

HARTLINE, H. K., \& RATLIFF, F. Spatial summation of inhibitory influences in the eye of the Limulus, and the mutual interaction of receptor units. Joumal of General Physiology, $1958,41,1049-1066$.

KAHNEMAN, D. An onset-onset law for one case of apparent motion and metacontrast. Perception \& Psychophysics, 1967, 2, 577-584.

KAHNEMAN, D. Method, findings, and theory in studies of visual masking. Psychological Bulletin, 1968, 70, 404-425.
KINSBOURNE, M.. \& WARRINGTON, E. The effect of an after-coming random pattern on the perception of brief visual stimuli. Quarterly Journal of Experimental Psychology, 1962a, 14, 223-234.

KINSBOURNE, M., \& WARRINGTON, l: Further studies on the masking of brief visual stimuli by a random pattern. Quarterly Journal of Experimental Psychology, 1962b, 14, 235-245.

KOLERS, P. A., \& ROSNER, B. S. On visual masking (metacontrast): Dichoptic observation. American Journal of Psychology $1960,73,2-21$.

MAYZNER, M. S., TRESSELT, M. E., \& HELFER, M. S. A provisional model of visual information processing with sequential inputs. Psychonomic Monograph Supplements, 1967. 2 (Whole No. 23), 91-108.

PARLEE, M. B. Visual backward masking of a single line by a single line. Vision Research, $1969,9,199-205$.

RAAB, D. H. Backward masking. Psychological Bulletin, 1963, 60, 118-129.

RATLIFF, F. Mach bands: Quantitattive studies on neural networks in the retine San Francisco: Holden-Day, 1965.

ROBINSON, $\mathrm{D}$. N. Disinhibition of visual masked stimuli. Science, 1966, 154, 157-158.

ROBINSON, D. N. Visual disinhibition with binocular and interocular presentations. Journal of the Optical Society of America, 1968, 58, 254-257.

ROTHBERG, J. M. Simulation of neural nets with some applications to visual information processing. Computers \& Biomedical Research, $1968,1,435-451$.

SCHILLER, P. H. Monoptic and dichoptic visual masking by pattems and flashes. Journal of Experimental Psychology, 1965, 69, 193-199.

SCHILLER, P. H. Single unit analysis of backward visual masking and metacontrast in the cat lateral geniculate nucleus. Vision Research, 1968, 8, 855-866.

SCHILLER, P. H., \& CHOROVER, S. L. Metacontrast: I ts relation to evoked potentials. Science, 1966, 153, 1398-1400.
SCHILLER, P. H., \& SMITH, M. C. Detection in metacontrast. Joumal of Fxperimental Psychology, 1966, 71, 32-39.

SEKULER, R. W. Spatial and temporal determinants of visual backward masking. Journal of Experimental Psychology, 1965. 70, 401-406.

UTTAL, W. R. Masking of alphabetic character recognition by dynamic visual noise (DVN). Perception \& Psychophysics, 1969a, 6, 121-128.

UTTAL, $W$. $R$. The character in the hole experiment. Perception \& Psychophysics, 1969b, 6, 177-181.

UTTAL, W. R. Masking of alphabetic character recognition by ultrahigh density dynamic visual noise. Perception \& Psychophysics, 1970a, 7, 19-22

UTTAL, W. R. Violations of visual simultaneity. Perception \& Psychophysics, 1970b, 7, 133-136.

von BÉKÉSY, G. Sensory inhibition. Princeton, N.J: Princeton University Press, 1967.

von BÉKÉSY, G. Mach- and Hering-type lateral inhibition in vision. Vision Research, 1968, 8, 1483-1499.

WEISSTEIN, N. A Rashevsky-Landahl neural net: Simulation of metacontrast. Psychological Review, 1968, 75, 494-521.

\section{NOTES}

1. This work was supported in part by a grant from the National Science Foundation, GB 12771

2. Address: Department of Psychology, The University of Michigan, Ann Arbor, Michigan 48104.

3. This difference may, in fact, be due to some occasional occlusion of the noise rather than to a peripheral interactive effect. If so, it would even further strengthen the conclusion that there is a central locus for the masking effect obtained with these dots.

(Accepted for publication October 8, 1969.) 